\title{
Triple Antithrombotic Therapy - Is It History or Should We Still Do It?
}

\author{
Diana Opincariu
}

University of Medicine, Pharmacy, Science and Technology, Târgu Mureș, Romania

\section{CORRESPONDENCE}

Diana Opincariu

Str. Gheorghe Marinescu nr. 38

540139 Târgu Mureș, Romania

Tel: +40 265215551

E-mail: diana.opincariu@yahoo.ro
Patients with atrial fibrillation (AF) typically require oral anticoagulants for the prevention of cardioembolic events, as the left atrium is an environment characterized by low shear stress. This low shear stress leads to the formation of thrombi that are not as platelet-dependent such as, for example, thrombi that form during an acute coronary syndrome, an event associated with high shear stress and platelet-rich thrombi. This explains the need for oral anticoagulants in AF patients and the need for dual antiplatelet therapy in coronary thrombosis. However, when a combination of the two is met (AF in patients undergoing PCI, or acute coronary events in AF patients), the so-called triple antithrombotic therapy emerges as a therapeutic indication, which also comes with its associated high bleeding risk. ${ }^{1,2}$

The latest therapeutic guidelines propose that individuals with atrial fibrillation, with a CHA2DS2-VASc score of minimum 2 for males and 3 for females, should be treated with oral anticoagulants, and that non-vitamin $\mathrm{K}$ antagonists (NOACs) are preferred over warfarin if there are no contraindications which include valvular AF, defined in the presence of moderate-to-severe mitral stenosis, or a mechanical cardiac valve. ${ }^{3}$

There is a continuously growing elderly population and the coexistence of AF with coronary artery disease (CAD), which will also increase the incidence of patients that require long-term oral anticoagulation. Furthermore, there is a high number of AF patients, up to $20-30 \%$, that will require percutaneous coronary revascularization, either during an elective procedure, or for an acute coronary syndrome (ACS). ${ }^{4}$ The implantation of a coronary stent brings about the clear indication for dual antiplatelet therapy with aspirin and a P2Y12 inhibitor (clopidogrel, ticagrelor, prasugrel, cangrelor), for a variable duration of time, for the prevention of secondary ischemic events and major adverse cardiac events (MACE). ${ }^{5}$

On the other hand, around 7\% of patients undergoing PCI with stent angioplasty present AF with indication of oral anticoagulation. The management of such patients is clinically challenging, as they present indication for both dual antiplatelet regimens as well as oral anticoagulation and are at high risk for 
both ischemic events (including stroke, stent thrombosis, coronary thrombosis) and hemorrhagic ones. ${ }^{6}$ Previous guideline recommendations in such cases were to prescribe triple antithrombotic therapy for a period of time, with aspirin, a P2Y12 inhibitor (clopidogrel; prasugrel or ticagrelor is to be avoided as part of the triple therapy due to greater risk of bleeding), and an oral anticoagulant (usually vitamin $\mathrm{K}$ antagonists), followed by a dual antithrombotic regimen (oral anticoagulant and a single antiplatelet). If NOACs were considered in this clinical scenario, the recommendation was to use the lowermost effective dose for the prevention of cerebrovascular cardioembolic events in AF patients. ${ }^{7}$

Several studies have been conducted in order to adjust current guideline recommendations in subjects who need both antiplatelet and anticoagulant therapies. Initial studies have investigated vitamin $\mathrm{K}$ antagonists (VKAs) together with single - or dual - antiplatelet therapies in AF subjects undergoing PCI.

The WOEST trial (What is the Optimal antiplatelet and anticoagulant therapy in patients with oral anticoagulation and coronary StenTing) investigated dual versus triple antithrombotic therapies in individuals with indication of oral anticoagulants ( $70 \%$ with $\mathrm{AF}$ ), who experienced coronary revascularization. The dual-therapy arm received clopidogrel and warfarin, while the triple-therapy arm added aspirin to the combination. Although the trial did not include a sufficient number of patients in order to evaluate the ischemic outcomes, the results showed that the dual-therapy group presented a lower incidence of major and non-major hemorrhagic episodes, lower one-year all-cause death, but no significantly different rates of myocardial infarction, ischemic stroke, or stent thrombosis compared to patients that had been assigned to the triple antithrombotic regimen. ${ }^{8}$

The ISAR-TRIPLE study (Triple Therapy in Patients on Oral Anticoagulation After Drug Eluting Stent Implantation) aimed to study the time length of triple antithrombotic treatment in subjects who receive oral anticoagulants and undergo percutaneous implantation of a coronary drug-eluting stent, as in such cases, there is a high risk of stent thrombosis and concomitant high bleeding risk. The study blindly assigned a total of 614 patients to group 1 ( 6 weeks of clopidogrel in association with aspirin and warfarin) and group 2 (6 months of triple therapy). Most included patients required oral anticoagulation for AF (82.7\% of group 1, $85 \%$ of group 2), followed by the presence of a mechanical heart valve or venous thromboembolic events. The results showed no difference between groups regarding the cumulative incidence of death, myo- cardial infarction, in-stent thrombosis, stroke, or major hemorrhagic episodes (HR 1.14, $\mathrm{p}=0.63$ ), but the BARC bleeding frequency was higher in the 6-months group ( $27.9 \%$ vs. $20.5 \%$, HR $0.68, \mathrm{p}=0.04$ ). However, the main study finding was that the reduction of triple therapy to 6 weeks was not superior to the 6-month regimen, regarding clinical net outcomes. ${ }^{9}$

Numerous large clinical studies have already established the safety and efficacy of NOACs for preventing ischemic cerebrovascular events in subjects with non-valvular AF, including the RELY trial (dabigatran $110 \mathrm{mg}$ and $150 \mathrm{mg}$ twice/day), the ROCKET-AF study (rivaroxaban $20 \mathrm{mg}$ daily), ARISTOTLE (apixaban $5 \mathrm{mg}$ twice/day), and the newest ENGAGE AF-TIMI 48 study (edoxaban $30 \mathrm{mg}$ and $60 \mathrm{mg}$ daily), all showing the superiority of NOAC to warfarin in terms of ischemic stroke, systemic embolism, or transient ischemic attacks compared to VKAs. ${ }^{10}$ In addition, three randomized open-label clinical studies have aimed to evaluate the use of NOACs in AF subjects who experience percutaneous coronary revascularization or ACS, in order to establish their use in this particular clinical setting, without increasing the bleeding risk.

The PIONEER AF-PCI trial aimed to investigate the safety and efficacy of rivaroxaban in combination with either one or two antiplatelet agents in AF patients undergoing PCI, by including 2,124 subjects with non-valvular $\mathrm{AF}$ and PCI stent placement, who were divided into 3 study arms: group 1 - Rivaroxaban $15 \mathrm{mg} /$ day + one P2Y12 inhibitor for 12 months; group 2 - Rivaroxaban 2.5 $\mathrm{mg}$ twice/day + dual antiplatelet therapy for 1, 6, and 12 months respectively; group 3 - VKA + dual antiplatelet therapy for 1,6 , and 12 months. A fact worth mentioning is that most patients received clopidogrel as a P2Y12 inhibitor (93.1\% of group 1; $93.7 \%$ of group $2 ; 96.3 \%$ of group 3). The bleeding rates were significantly lower for patients assigned to group 1 and group 2 respectively, compared to patients who received triple antithrombotic therapy ( $16.8 \%$ for group 1 vs. $18.0 \%$ for group 2 vs. $26.7 \%$ for group $3, \mathrm{p}<0.001$ ), while the rates of cardiovascular deaths, myocardial infarction, and stroke did not differ significantly among the three study arms (6.5\% for group $1 \mathrm{vs.}$ $5.6 \%$ for group 2 vs. $6.0 \%$ for group $3, \mathrm{p}>0.05) .{ }^{11}$

REDUAL-PCI was a randomized study that had investigated dual therapy with dabigatran compared to triple therapy with warfarin in AF subjects undergoing PCI. The study included 2,725 patients that were blindly assigned to dual therapy: dabigatran $110 \mathrm{mg}$ twice/day $(\mathrm{n}=981)$ or $150 \mathrm{mg}$ twice/day $(\mathrm{n}=763)$ with a P2Y12 inhibitor, or to triple therapy: warfarin + a P2Y12 inhibitor + aspirin $(\mathrm{n}=981)$. In the dual-therapy group, study subjects were 
administered clopidogrel or ticagrelor in association with two doses of dabigatran, while in the triple-therapy group, participants were administered warfarin adjusted for an INR of 2.0-3.0, clopidogrel or ticagrelor, and aspirin (for one month if bare metal stents were implanted, 3 months if drug-eluting stents were used). The results revealed that the rate of major and clinically relevant bleeding events were significantly lower for subjects assigned to the dualtherapy regimen $(15.4 \%$ vs. $26.9 \%$, p for non-inferiority $<0.001$ ), while even the higher dose regimen for dabigatran was less likely to cause significant bleeding compared to the triple-therapy regimen ( $20.2 \%$ vs. $25.7 \%$, p for noninferiority $<0.001)$. And while being associated with a significantly lower bleeding risk, dabigatran dual therapy was comparable to the triple warfarin therapy regarding the rate of composite end-point of ischemic events (coronary, cerebrovascular, or systemic ischemia). ${ }^{12,13}$

AUGUSTUS is the latest trial with published results in March 2019, its primary aim being to shed some light on the still unclear matter of choosing the right antithrombotic regimen for patients who require oral anticoagulants for various clinical reasons, but who also undergo coronary percutaneous revascularization, thus needing antiplatelet therapies as well. This phase IV randomized study included 4,614 non-valvular AF subjects from 33 countries that had suffered an ACS or had undergone elective PCI for significant $\mathrm{CAD}$. The primary outcome measure included the rate of major or clinically relevant non-major bleeding during the treatment period with either apixaban or a VKA, or with aspirin versus no-aspirin regimen. The study population had been divided into two major groups: group 1 - apixaban $5 \mathrm{mg} / 2.5 \mathrm{mg}$ twice/day versus group 2 - a VKA once daily for INR 2.0-3.0. The subjects were further assigned to receive either placebo once daily, or aspirin $81 \mathrm{mg}$ once daily. ${ }^{14} \mathrm{~A}$ majority of $92.6 \%$ of patients included in the study received clopidogrel as the main antiplatelet treatment following PCI, while a total of $37.3 \%$ had suffered an ACS who underwent PCI, $23.9 \%$ had ACS and were managed pharmacologically, and $38.8 \%$ had undergone elective PCI. The results showed that during the 6-month follow-up, the apixaban group presented significantly lower rates of major bleeding events than the VKA group (10.5\% vs. $14.7 \%$, p for non-inferiority $<0.001$, p for superiority $<0.001$; HR: 0.69 , 95\% CI: 0.58-0.81). Concomitantly, patients receiving aspirin presented significantly higher rates of major or clinically relevant non-major bleeding compared to patients that had been assigned to placebo ( $16.1 \%$ vs. $9.0 \%$, HR: $1.89 \%$, $95 \%$ CI: $1.59-2.24, \mathrm{p}<0.001)$. The highest rate of hemorrhages was recorded among subjects who had received a VKA and aspirin (18.7\%), while the lowest was recorded in the apix- aban-placebo group (7.3\%). The rates of hospitalization or death were higher in the VKA group ( $57.2 \%$ vs. $69.2 \%$, HR $0.83, p=0.002)$ compared to apixaban, while the rates of death or ischemic events were not different between the two anticoagulant groups (14.3\% for apixaban vs. $15.3 \%$ for VKA, $\mathrm{p}>0.05)$. The antiplatelet group showed no significant difference between aspirin vs. placebo regarding the death and hospitalization outcome measure (65.7\% for aspirin vs. $60.6 \%$ for placebo, $\mathrm{p}>0.05$ ), while the rates of death and ischemic events were not assessed for this particular study population. ${ }^{15}$

Current clinical trial data suggest that less is more in the context of the antithrombotic regimens of subjects with non-valvular atrial fibrillation who undergo coronary PCI, either electively or during an acute coronary event. The risk of bleeding decreases significantly with simpler antithrombotic therapeutic schemes, with apparently no concomitant elevation of the ischemic risk. However, not all trials have studied the subsequent risk for coronary ischemic events or in-stent thrombosis, which strongly indicates the need for further trials on larger patient cohorts in order to establish this hazard. All in all, there is a trend towards choosing non-vitamin $\mathrm{K}$ antagonists for the prevention of cardioembolic risk in AF subjects with coronary stenting procedures, but still there is scarce data on their association with newer-generation P2Y12 inhibitors such as ticagrelor, which is currently prescribed at a large scale in acute coronary syndromes, as well as a lack of long-term follow-up of over a year in these patients, thus indicating the need for further research on the matter.

\section{CONFLICT OF INTEREST}

Nothing to declare.

\section{REFERENCES}

1. Capodanno D, Angiolillo DJ. Triple Antithrombotic Therapy at the Intercept Between Threats and Opportunities: Don't Throw Out the Baby With the Bath Water. JACC Cardiovasc Interv. 2017;10:1086-1088.

2. Lamberts $\mathrm{M}$, Olesen JB, Ruwald $\mathrm{MH}$, et al. Bleeding after initiation of multiple antithrombotic drugs, including triple therapy, in atrial fibrillation patients following myocardial infarction and coronary intervention: a nationwide cohort study. Circulation. 2012;126:1185-1193.

3. January CT, Wann LS, Calkins $H$, et al. 2019 AHA/ACC/HRS Focused Update of the 2014 AHA/ACC/HRS Guideline for the Management of Patients With Atrial Fibrillation: A Report of the American College of Cardiology/American Heart Association Task Force on Clinical Practice Guidelines and the Heart Rhythm Society. J Am Coll Cardiol. 2019. pii: S0735-1097(19)30209-8.

4. Mantis C, Alexopoulos D. Antithrombotic treatment in atrial fibrillation patients undergoing $\mathrm{PCl}$ : Is dual therapy the winner? Thrombosis Research. 2019;176:133-139.

5. Valgimigli M, Bueno H, Byrne RA, et al. 2017 ESC focused update on dual antiplatelet therapy in coronary artery disease developed in collaboration with EACTS. Eur Heart J. 2018;39:213-254 
6. Senoo K, Lane D, Lip GY. Stroke and Bleeding Risk in Atrial Fibrillation. Korean Circ J. 2014;44:281.

7. Kirchhof P, Benussi S, Kotecha D, et al. 2016 ESC Guidelines for the management of atrial fibrillation developed in collaboration with EACTS. Eur J Cardiothorac Surg. 2015;50:e1-e88.

8. Dewilde WJ, Oirbans T, Verheugt FW, et al. Use of clopidogrel with or without aspirin in patients taking oral anticoagulant therapy and undergoing percutaneous coronary intervention: an open-label, randomised, controlled trial. Lancet. 2013;381:1107-1115.

9. Fiedler KA, Maeng M, Mehilli J, et al. Duration of Triple Therapy in Patients Requiring Oral Anticoagulation After Drug-Eluting Stent Implantation: The ISAR-TRIPLE Trial. J Am Coll Cardiol. 2015;65:1619-1629.

10. Hammersley D, Signy M. Navigating the choice of oral anticoagulation therapy for atrial fibrillation in the NOAC era. Ther Adv Chronic Dis. 2017:8:165-176
11. Gibson CM, Mehran R, Bode C, et al. Prevention of Bleeding in Patients with Atrial Fibrillation Undergoing PCI. N Engl J Med. 2016;375:2423-2434.

12. Oldgren J, Steg PG, Hohnloser SH, et al. Dabigatran dual therapy with ticagrelor or clopidogrel after percutaneous coronary intervention in atrial fibrillation patients with or without acute coronary syndrome: a subgroup analysis from the RE-DUAL PCI trial. Eur Heart J. 2019. pii: ehz059.

13. Cannon CP, Bhatt DL, Oldgren J, et al. Dual Antithrombotic Therapy with Dabigatran after PCl in Atrial Fibrillation. N Engl J Med. 2017;377:1513-1524.

14. Lopes RD, Vora AN, Liaw D, et al. An open-label, $2 \times 2$ factorial, randomized controlled trial to evaluate the safety of apixaban vs. vitamin $\mathrm{K}$ antagonist and as $p$ pirin vs. placebo in patients with atrial fibrillation and acute coronary syndrome and/or percutaneous coronary intervention: rationale and design of the AUGUSTUS trial. Am Heart J. 2018;200:17-23.

15. Lopes RD, Heizer G, Aronson R, et al. Antithrombotic Therapy after Acute Coronary Syndrome or $\mathrm{PCl}$ in Atrial Fibrillation. N Engl J Med. 2019;380:1509-1524. 